\title{
Incremental Twisting Fault Tolerant Control for Hypersonic Vehicles With Partial Model Knowledge
}

\author{
Tuo Han, Qinglei Hu, Hyo-Sang Shin, Antonios Tsourdos, and Ming Xin
}

\begin{abstract}
A passive fault tolerant control scheme is proposed for the full reentry trajectory tracking of a hypersonic vehicle in the presence of modelling uncertainties, external disturbances, and actuator faults. To achieve this goal, the attitude error dynamics with relative degree two is formulated first by ignoring the nonlinearities induced by the translational motions. Then, a multivariable twisting controller is developed as a benchmark to ensure the precise tracking task. Theoretical analysis with the Lyapunov method proves that the attitude tracking error and its first-order derivative can simultaneously converge to the origin exponentially. To depend less on the model knowledge and reduce the system uncertainties, an incremental twisting fault tolerant controller is derived based on the incremental nonlinear dynamic inversion control and the predesigned twisting controller. It is shown that not only the benefits of both incremental control and twisting control are inherited, but also their side effects are reduced. Notably, the proposed controller is user friendly in that only fixed gains and partial model knowledge are required. Numerical simulations in various cases and comparison studies are conducted to verify the effectiveness of the proposed method.
\end{abstract}

Index Terms-Hypersonic vehicle, reentry trajectory tracking, fault tolerant control, twisting control, actuator faults.

\section{INTRODUCTION}

$\mathrm{S}$ AFETY has been playing an important role in the aerospace industry and is demanding for the flight control of hypersonic vehicles due to its complex structure, civil/military mission, and high cost. The main factors that threaten flight safety are the actuator faults, unmodelled dynamics, and external disturbances [1,2]. To handle this issue, the fault tolerant control (FTC) has emerged and witnessed a wide range of applications in robots [3], ground vehicles [4], aerial vehicles [5], and spacecraft [6].

Generally, FTC can be classified into two groups: active FTC and passive FTC [7]. The active FTC framework estimates faults via the fault detection and identification (FDI) method. Then, it reconfigures the controller for self-healing utilizing the

Manuscript received Month xx, 20xx; revised Month xx, xxxx; accepted Month $\mathrm{xx}, 20 \mathrm{xx}$. This work was supported by the National Natural Science Foundation of China (No. 61960206011, No. 61633003). (Corresponding author: Hyo-Sang Shin)

T. Han and Q. Hu (co-first author) are with the School of Automation Science and Electrical Engineering, Beihang University, Beijing, 100191, China (E-mail: hantuo@buaa.edu.cn; huql_buaa@buaa.edu.cn)

H. Shin and A. Tsourdos are with the School of Aerospace, Transport an Manufacturing, Cranfield University, Cranfield, MK43 OAL, U.K. (E-mail: h.shin@cranfield.ac.uk; a.tsourdos@cranfield.ac.uk).

M. Xin is with the Department of Mechanical and Aerospace Engineering, University of Missouri, Columbia, MO 65211, USA (E-mail: xin@missouri.edu). remaining healthy actuators. For instance, the fault efficiency factor was estimated by an adaptive composite observer in [4] and combined with the fast terminal sliding mode to obtain an active FTC for electric vehicles. In [5], a super twisting observer was utilized to estimate the servo's stuck fault of an unmanned aerial vehicle (UAV) and an integral FTC law was developed. The active FTC of near-space hypersonic vehicles was studied in [8] via a sliding mode observer-based FDI. In [9], a neural network adaptive FDI process was combined with the nonlinear dynamic inversion (NDI) control to actively compensate for the actuator faults of UAV. To deal with the undetected/missed small actuator faults, an adaptive FDI that only detects but not estimates the fault was integrated within a robust UAV control framework [10]. The total effect of the actuator fault was estimated in [11] to develop an active FTC for spacecraft under input saturation. The key of active FTC is to enhance system robustness relying on the FDI technique that requires more about the model knowledge.

The passive FTC scheme preserves/enhances the system robustness against faults and uncertainties without using FDI. For example, the nonlinear disturbance observer was combined with sliding mode control (SMC) in [2] for the hypersonic vehicle FTC issue. A prescribed-time FTC law was designed in [3] by integrating a barrier Lyapunov function. Based on the efficiency of SMC, a passive FTC law for spacecraft was developed in [6] using the time-varying SMC. Besides, various passive FTC laws were also developed for hypersonic vehicles based on the terminal SMC $[12,13]$, barrier-Lyapunov function [14], and the singular perturbation-based control [15]. The merits of the passive FTC are that it can improve computational efficiency compared to the active FTC.

Although the above success has been achieved in FTC, a key problem remains open in this field. Specifically, the FTC will become less robust with either poor FDI performance for the active FTC or inaccurate uncertainty cancellation for the passive FTC. However, if more accurate FDI or uncertainty compensation methods are used, the computational complexity will increase and much more effort will be required to obtain the accurate model knowledge. Notably, the state-of-the-art FTC strategies face the following design difficulties: $i$ ) The strong dependency on the model knowledge; ii) System uncertainty increases with less model knowledge; iii) Greater minimum control gains are required under larger uncertainty magnitude; iv) Severe chattering will occur with larger control gains for SMC-based FTC. It is difficult and contradictory to 
meet the objectives of reduced uncertainty, less model dependency, smaller control gains, and less chattering phenomenon simultaneously.

To handle such a dilemma, the incremental nonlinear dynamic inversion (INDI) was proposed in [16] and has attracted much attention for the FTC design in the aerospace community $[17,18,19]$. Recent flight tests $[20,21]$ have also demonstrated the applicability and efficiency of this method. The main advantage of INDI is that not only the model dependency is reduced, but also the robustness is improved. Recently, the combination of INDI and SMC (named INDI-SMC $[18,19,22])$ that inherits the merits and avoids the defects of both methods has been studied to further improve the robustness of INDI. Motivated by the above mentioned, this paper proposes an incremental twisting FTC law that innovatively integrates the INDI and twisting control $[23,24]$ (a type of second-order SMC) for the full reentry trajectory tracking of hypersonic vehicles. Note that it is more challenging and significant to deal with the full trajectory tracking issue because of the unknown and long-duration flight environment, time-varying aerodynamics, nonlinear dynamics, strong couplings, and especially in the presence of uncertainties, disturbances, and actuator faults. The main contributions of this paper are as follows.

i) Contributions to the twisting control: A multivariable twisting controller is proposed for systems with relative degree two. It is shown that the system tracking error and its derivative can exponentially converge to the origin simultaneously.

ii) Contributions to the INDI architecture: The INDI is extended with twisting control (named as INDI-TW) to further enhance its robustness. Moreover, the INDI-TW has a simple structure without requiring the design of sliding surfaces.

iii) Contributions to the FTC of hypersonic vehicles: The INDI-TW is applied to the FTC for hypersonic vehicles with partial model knowledge under system uncertainties, external disturbances, and actuator faults. Unlike most FTC designs for hypersonic vehicles that only consider a fixed feature point or the longitudinal dynamics, the proposed technique is feasible in tracking a full reentry trajectory with fixed gains.

The rest of this paper is organized as follows. The problem is formulated in Section II. The twisting control based on NDI (NDI-TW) and INDI (INDI-TW) is presented in Section III, and the performance of the proposed method is verified in Section IV. Finally, conclusions are drawn in Section V.

\section{MOdEl DESCRIPTION AND PROBLEM Formulation}

In this section, the robust tracking problem for an aerodynamic surface actuated hypersonic vehicle that behaves bank-to-turn (BTT) maneuvers is formulated under external disturbances, model uncertainties, and actuator faults.

\section{A. Nonlinear Model for Hypersonic Vehicles}

The nonlinear six-degree-of-freedom model for hypersonic vehicles can be presented as [25-27]

i) Translational Equations:

$$
\begin{gathered}
\dot{h}=V \sin \gamma \\
\dot{\phi}=V \cos \gamma \sin \vartheta /\left[\left(r_{e}+h\right) \cos \theta\right] \\
\dot{\theta}=V \cos \gamma \cos \vartheta /\left(r_{e}+h\right)
\end{gathered}
$$

$$
\begin{aligned}
\dot{V}= & \omega_{e}^{2}\left(r_{e}+h\right) \cos \theta(\sin \gamma \cos \theta-\cos \gamma \sin \theta \cos \vartheta)-D / m-g \sin \gamma \\
\dot{\vartheta}= & L \sin \sigma /(m V \cos \gamma)+V \cos \gamma \sin \vartheta \tan \theta /\left(r_{e}+h\right) \\
& +\omega_{e}^{2}\left(r_{e}+h\right) \sin \theta \cos \theta \sin \vartheta / V \cos \gamma-2 \omega_{e}(\tan \gamma \cos \theta \cos \vartheta-\sin \theta) \\
\dot{\gamma}= & L \cos \sigma /(m V)-g \cos \gamma / V+V \cos \gamma /\left(r_{e}+h\right)+2 \omega_{e} \cos \theta \sin \vartheta \\
& +\omega_{e}^{2}\left(r_{e}+h\right) \cos \theta(\cos \gamma \cos \theta+\sin \gamma \sin \theta \cos \vartheta) / V
\end{aligned}
$$

where $h$ is the flight altitude, $V$ is the vehicle's earth relative velocity, $\gamma$ is the flight path angle, $\vartheta$ is the heading angle, and $\sigma$ is the bank angle. $\phi$ and $\theta$ are the latitude and longitude, respectively. $r_{e}, \omega_{e}, g$ denote the radius, angular speed, and gravity constant of the Earth, respectively. $L, D, m$ are the lift force, drag force, and mass of the vehicle, respectively. Note that the kinematics (1) (6) is based on the zero sideslip angle assumption due to the BTT maneuver requirement.

ii) Rotational Equations:

$$
\begin{gathered}
\dot{\alpha}=q-p \cos \alpha \tan \beta+r \sin \alpha \tan \beta+\sin \sigma / \cos \beta[\dot{\vartheta} \cos \theta \\
\left.-\dot{\phi} \sin \vartheta \sin \gamma+\left(\dot{\theta}+\omega_{e}\right)(\cos \phi \cos \vartheta \sin \gamma-\sin \phi \cos \gamma)\right] \\
-\cos \sigma / \cos \beta\left[\dot{\gamma}-\dot{\phi} \cos \vartheta-\left(\dot{\theta}+\omega_{e}\right) \cos \phi \sin \vartheta\right] \\
\dot{\beta}=p \sin \alpha+\cos \sigma\left[\dot{\vartheta} \cos \gamma-\dot{\phi} \sin \vartheta \sin \gamma-\left(\dot{\theta}+\omega_{e}\right)(\cos \phi \cos \vartheta \sin \gamma\right. \\
-\sin \phi \cos \gamma)]+\sin \sigma\left[\dot{\gamma}-\dot{\phi} \cos \vartheta+\left(\dot{\theta}+\omega_{e}\right) \cos \phi \sin \vartheta\right]+r \cos \alpha \\
\dot{\sigma}=-p \cos \alpha \cos \beta-q \sin \beta-r \sin \alpha \cos \beta+\dot{\alpha} \sin \beta-\dot{\vartheta} \sin \gamma \\
-\dot{\phi} \sin \vartheta \cos \gamma+\left(\dot{\theta}+\omega_{e}\right)(\cos \phi \cos \vartheta \cos \gamma+\sin \phi \sin \gamma) \\
\dot{p}=a_{1} M_{r}+a_{2} M_{y}+a_{3} p q+a_{4} q r \\
\dot{q}=a_{5} M_{p}+a_{6}\left(r^{2}-p^{2}\right)+a_{7} p r \\
\dot{r}=a_{2} M_{r}-a_{3} q r+a_{8} M_{y}+a_{9} p q
\end{gathered}
$$

where $\left[a_{5}, a_{6}, a_{7}\right]=\left[1, I_{x z}, I_{z z}-I_{x x}\right] / I_{y y}$, and $\left[a_{1}, a_{2}, a_{3}, a_{4}, a_{8}, a_{9}\right]=$ $\left[I_{z z}, I_{x z},\left(I_{x x}-I_{y y}+I_{z z}\right) I_{x z},\left(I_{y y}-I_{z z}\right) I_{z z}-I_{x z}^{2}, I_{x x},\left(I_{x x}-I_{y y}\right) I_{x x}+I_{x z}^{2}\right] / I_{x x} I_{z z}-I_{x z}^{2}$. $\alpha$ is the angle of attack (AOA), $\beta$ is the sideslip angle. $M_{r}, M_{p}, M_{y}$ are the roll, pitch, and yaw moments, $p, q, r$ are the corresponding roll, pitch, and yaw angular rates, respectively. $I_{i j}(i, j=x, y, z)$ denote the moment of inertia.

\section{B. Aerodynamic Force and Moment Model}

The aerodynamic lift and drag forces are presented as

$$
\left[\begin{array}{l}
L \\
D
\end{array}\right]=\left[\begin{array}{l}
\bar{q} C_{L}\left(M a, \alpha, \delta_{a}, \delta_{e}, \delta_{r}\right) S_{r e f} \\
\bar{q} C_{D}\left(M a, \alpha, \delta_{a}, \delta_{e}, \delta_{r}\right) S_{r e f}
\end{array}\right]
$$

where $\bar{q}=0.5 \rho V^{2}$ is the dynamic pressure, $\rho$ is the atmospheric density, $S_{r e f}$ is the reference area. $C_{L}, C_{D}$ are lift and drag coefficients related to the Mach number $(M a)$, AOA, and fin deflections ( $\delta_{a}$ the aileron, $\delta_{e}$ the elevator, and $\delta_{r}$ the rudder).

The roll, pitch, and yaw moments are computed by

$$
\left[\begin{array}{c}
M_{r} \\
M_{p} \\
M_{y}
\end{array}\right]=\left[\begin{array}{l}
\bar{q} m_{r}\left(M a, \alpha, \beta, \delta_{a}, \delta_{e}, \delta_{r}\right) S_{r e f} L_{r e f} \\
\bar{q} m_{p}\left(M a, \alpha, \delta_{a}, \delta_{e}, \delta_{r}\right) S_{r e f} L_{r e f} \\
\bar{q} m_{y}\left(M a, \alpha, \beta, \delta_{a}, \delta_{e}, \delta_{r}\right) S_{r e f} L_{r e f}
\end{array}\right]
$$

where $L_{r e f}$ is the reference length, $m_{r}, m_{p}, m_{y}$ are the roll, pitch, and yaw moment coefficients related to the Mach number, AOA, sideslip angle, and fin deflections.

C. Attitude Error Dynamics with Uncertainties, Disturbances, and Actuator Faults

To design a proper attitude controller such that the hypersonic vehicle can precisely track the reference trajectory 
generated by (1) (6), the attitude dynamics is presented in a compact form from $(7) \sim(12)$ as follows

$$
\left\{\begin{array}{l}
\dot{\boldsymbol{\Psi}}=\boldsymbol{T} \boldsymbol{\omega} \\
\dot{\boldsymbol{\omega}}=-\boldsymbol{I}^{-1} \boldsymbol{\Gamma} \boldsymbol{\omega} \boldsymbol{\omega}+\boldsymbol{I}^{-1} \boldsymbol{M}
\end{array}\right.
$$

where $\boldsymbol{\Psi}=[\alpha, \beta, \sigma]^{T}, \boldsymbol{\omega}=[p, q, r]^{T}, \boldsymbol{M}=\left[M_{r}, M_{p}, M_{y}\right]^{T}$, and

$$
\begin{gathered}
\boldsymbol{T}=\left[\begin{array}{ccc}
-\cos \alpha \tan \beta & 1 & \sin \alpha \tan \beta \\
\sin \alpha & 0 & \cos \alpha \\
-\cos \alpha \cos \beta & -\sin \beta & -\sin \alpha \cos \beta
\end{array}\right], \\
\boldsymbol{I}=\left[\begin{array}{ccc}
I_{x x} & -I_{x y} & -I_{x z} \\
-I_{y x} & I_{y y} & -I_{y z} \\
-I_{z x}-I_{z y} & I_{z z}
\end{array}\right], \boldsymbol{\Gamma}=\left[\begin{array}{ccc}
0-r & q \\
r & 0 & -p \\
-q & p & 0
\end{array}\right]
\end{gathered}
$$

Note that (13) neglects the translational part and the Earth's angular speed in (7) (9). Such manipulation is reasonable since the translational motion is much slower than the rotational motion for hypersonic vehicles [24]. In practice, to attain the specific control surface deflections, the aerodynamic moment vector $\boldsymbol{M}$ is further presented as

\section{$\boldsymbol{M}=\boldsymbol{P C} \boldsymbol{u}$}

where $\boldsymbol{u}=\left[\delta_{1}, \delta_{2}, \delta_{3}, \cdots \delta_{p}\right]^{T}, p \geq 3$ is the actual control surface deflection vector, $\boldsymbol{C} \in \boldsymbol{R}^{3 \times p}$ is the control allocation matrix whose elements are $c_{i j} \in[0,1](i=1,2,3, j=1,2,3, \cdots p)$, and

$$
\boldsymbol{P}=\bar{q} S_{r e f} L_{r e f}\left[\begin{array}{ccc}
m_{r a} & m_{r e} & m_{r r} \\
m_{p a} & m_{p e} & m_{p r} \\
m_{y a} & m_{y e} & m_{y r}
\end{array}\right]
$$

where $m_{i j}(i=r, p, y, j=a, e, r)$ denote the coefficients related to the partial differential terms of $m_{i}$ with respect to $\delta_{a}, \delta_{e}, \delta_{r}$.

Considering the reference command as $\boldsymbol{\Psi}_{c}=\left[\alpha_{c}, \beta_{c}, \sigma_{c}\right]^{T}$ and defining the attitude tracking error as $\boldsymbol{e}_{\psi}=\boldsymbol{\Psi}-\boldsymbol{\Psi}_{c}$, the attitude error dynamics can be obtained from (13),(14) as

$$
\left\{\begin{array}{l}
\dot{\boldsymbol{e}}_{\psi}=\boldsymbol{e}_{\omega} \\
\dot{\boldsymbol{e}}_{\omega}=\overline{\boldsymbol{A}}+\overline{\boldsymbol{B}} \boldsymbol{u}-\ddot{\boldsymbol{\Psi}}_{c}+\overline{\boldsymbol{\Omega}}
\end{array}\right.
$$

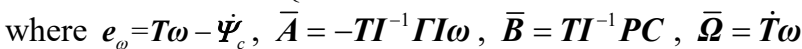

Remark 1. $\alpha_{c}, \sigma_{c}$ are obtained by optimizing (1) (6) with terminal and process constraints. $\beta_{c}$ is set to zero due to the BTT maneuver. Tracking of these angles is a common objective in hypersonic vehicle control problems [13,24-27].

Assumption 1. The reference command $\Psi_{c}$ is bounded, twice differentiable and smooth, with bounded $\dot{\Psi}_{c}$ and $\ddot{\Psi}_{c}$.

To further consider the practical situation, the modelling uncertainties, external disturbances, and actuator faults are involved in the second equation of (15). Then, (15) becomes

$$
\left\{\begin{array}{l}
\dot{\boldsymbol{e}}_{\psi}=\boldsymbol{e}_{\omega} \\
\dot{\boldsymbol{e}}_{\omega}=\boldsymbol{A}+\boldsymbol{B} \boldsymbol{u}-\ddot{\boldsymbol{\Psi}}_{c}+\boldsymbol{\Omega}
\end{array}\right.
$$

where $\boldsymbol{\Omega}$ is the lumped external disturbance vector, and

$$
\begin{gathered}
\boldsymbol{A}=\overline{\boldsymbol{A}}+\left(\boldsymbol{A}_{F}-\overline{\boldsymbol{A}}\right) \delta(t)+\Delta \boldsymbol{A} \\
\boldsymbol{B}=\overline{\boldsymbol{B}}+\left(\boldsymbol{B}_{F}-\overline{\boldsymbol{B}}\right) \delta(t)+\Delta \boldsymbol{B} \\
\boldsymbol{\Omega}=\overline{\boldsymbol{\Omega}}+\boldsymbol{d}
\end{gathered}
$$

where $\Delta \boldsymbol{A}, \Delta \boldsymbol{B}$ are unmodeled dynamics, $\boldsymbol{A}_{F}, \boldsymbol{B}_{F}$ are versions of $\overline{\boldsymbol{A}}, \overline{\boldsymbol{B}}$ under actuator faults, $\boldsymbol{d}$ is the external disturbance, $\delta(t)$ is a function that triggers the fault at $t=t_{F}$ and is described as

$$
\delta(t)= \begin{cases}0, & \text { if } t<t_{F} \\ 1, & \text { if } t \geq t_{F}\end{cases}
$$

Assumption 2. The lumped disturbance $\boldsymbol{\Omega}$ is bounded.

\section{Control Objectives}

i) Stabilize the attitude error dynamics (16) such that $\boldsymbol{e}_{\psi} \rightarrow 0$, i.e., the following aims should be achieved

$$
\alpha \rightarrow \alpha_{c}, \beta \rightarrow \beta_{c}, \sigma \rightarrow \sigma_{c}
$$

ii) Design the control law $\boldsymbol{u}$ using partial model knowledge, i.e., the uncertainties $\Delta \boldsymbol{A}, \Delta \boldsymbol{B}$, fault information $\boldsymbol{A}_{F}, \boldsymbol{B}_{F}, \delta(t)$, disturbance $\boldsymbol{\Omega}$, and even the nominal system dynamics $\overline{\boldsymbol{A}}$, are unknown and will not be compensated via the estimation tools.

\section{INDI-TW CONTROL SCHEME}

To achieve the control objectives presented in Sec. II.D, the NDI-TW is first designed and analyzed as a benchmark. Then, the INDI-TW is proposed and its stability is proved. The comparison between NDI-TW and INDI-TW is analyzed.

\section{A. NDI-TW Controller Design and Analysis}

For the attitude error dynamics (16) with relative degree two, a multivariable twisting controller inspired by [28] can be designed based on the NDI control structure as follows

$$
\boldsymbol{u}_{n d i-t w}=\overline{\boldsymbol{B}}^{+} \underbrace{\left(-\overline{\boldsymbol{A}}+\ddot{\boldsymbol{\Psi}}_{c}\right)}_{\boldsymbol{u}_{e q}}+\overline{\boldsymbol{B}}^{+} \underbrace{\left(-k_{1} \frac{\boldsymbol{e}_{\psi}}{\left\|\boldsymbol{e}_{\psi}\right\|}-k_{2} \frac{\boldsymbol{e}_{\omega}}{\left\|\boldsymbol{e}_{\omega}\right\|}-k_{3} \boldsymbol{e}_{\psi}-k_{4} \boldsymbol{e}_{\omega}\right)}_{u_{w}} \text { (21) }
$$

where $k_{1}, k_{2}, k_{3}, k_{4}$ are positive gains to be designed, the pseudoinverse matrix $\overline{\boldsymbol{B}}^{+}=\overline{\boldsymbol{B}}^{T}\left(\overline{\boldsymbol{B}} \overline{\boldsymbol{B}}^{T}\right)^{-1}$ and satisfies $\overline{\boldsymbol{B}} \overline{\boldsymbol{B}}^{+}=\boldsymbol{I}_{3}$. Substituting (21) into (16) leads to

$$
\left\{\begin{array}{l}
\dot{\boldsymbol{e}}_{\Psi}=\boldsymbol{e}_{\omega} \\
\dot{\boldsymbol{e}}_{\omega}=\boldsymbol{A}+\boldsymbol{B}\left(\overline{\boldsymbol{B}}^{+} \boldsymbol{u}_{e q}+\overline{\boldsymbol{B}}^{+} \boldsymbol{u}_{t w}\right)-\ddot{\boldsymbol{\Psi}}_{c}+\boldsymbol{\Omega}
\end{array}\right.
$$

Considering that

$$
\begin{aligned}
\dot{\boldsymbol{e}}_{\omega} & =\boldsymbol{A}+\boldsymbol{B} \overline{\boldsymbol{B}}^{+} \boldsymbol{u}_{e q}+\boldsymbol{B} \overline{\boldsymbol{B}}^{+} \boldsymbol{u}_{t w}-\ddot{\boldsymbol{\Psi}}_{c}+\boldsymbol{\Omega}-\overline{\boldsymbol{A}}+\ddot{\boldsymbol{\Psi}}_{c}-\left(-\overline{\boldsymbol{A}}+\ddot{\boldsymbol{\Psi}}_{c}\right)+\boldsymbol{u}_{t w}-\boldsymbol{u}_{t w} \\
& =\boldsymbol{u}_{t w}+\boldsymbol{A}-\overline{\boldsymbol{A}}+\boldsymbol{B} \overline{\boldsymbol{B}}^{+} \boldsymbol{u}_{e q}+\boldsymbol{B} \overline{\boldsymbol{B}}^{+} \boldsymbol{u}_{t w}+\boldsymbol{\Omega}-\boldsymbol{u}_{e q}-\boldsymbol{u}_{t w}
\end{aligned}
$$

(22) can be rewritten as

$$
\left\{\begin{array}{l}
\dot{\boldsymbol{e}}_{\Psi}=\boldsymbol{e}_{\omega} \\
\dot{\boldsymbol{e}}_{\omega}=\boldsymbol{u}_{t w}+\boldsymbol{\varepsilon}_{n d i-t w}
\end{array}\right.
$$

where

$$
\boldsymbol{\varepsilon}_{n d i-t w}=\boldsymbol{A}-\overline{\boldsymbol{A}}+\left(\boldsymbol{B} \overline{\boldsymbol{B}}^{+}-\boldsymbol{I}\right)\left(\boldsymbol{u}_{e q}+\boldsymbol{u}_{t w}\right)+\boldsymbol{\Omega}
$$

represents the uncertainty residual existing in the error dynamics (16) under the NDI-TW controller (21).

Assumption 3. The residual $\boldsymbol{\varepsilon}_{\text {ndi-tw }}$ presented in (24) is bounded and satisfies $\left\|\boldsymbol{\varepsilon}_{n d i-t w}\right\| \leq \chi$ with $\chi$ a positive scalar .

Remark 2. Assumption 3 is also a primary hypothesis in many robust control methods (e.g. $[1,2,6,11,14,24,27]$, to name a few).

Theorem 1. For the attitude error dynamics (16) (or the transformed (23)), the NDI-TW controller (21) can guarantee the simultaneous convergence of $\boldsymbol{e}_{\psi}$ and $\boldsymbol{e}_{\omega}$ exponentially under Assumption 3 if the gains in (21) satisfy

$$
\left\{\begin{array}{l}
k_{1} \geq k_{2}+\chi+\varsigma_{1} \\
k_{2} \geq(2 \bar{m}-1) \chi+(\bar{m}-1) \varsigma_{1}+\varsigma_{2}
\end{array}\right.
$$




$$
\left[\begin{array}{cc}
2 \bar{m} k_{3} & k_{3}(1-1 / \bar{m}) \\
* & 2\left(k_{4}-\bar{m}\right)
\end{array}\right]>0
$$

for some $\bar{m}>1, \varsigma_{1}>0, \varsigma_{2}>0$.

Proof. It will first be shown that the equilibrium $\boldsymbol{e}_{\psi}=\boldsymbol{e}_{\omega}=0$ is a unique point of the closed-loop system (23). Specifically, the error dynamics with $\boldsymbol{e}_{\omega}=0$ can be described as [28]

$$
\left\{\begin{array}{l}
\dot{\boldsymbol{e}}_{\Psi}=0 \\
\dot{\boldsymbol{e}}_{\omega}=-k_{1} \frac{\boldsymbol{e}_{\psi}}{\left\|\boldsymbol{e}_{\Psi}\right\|}-k_{3} \boldsymbol{e}_{\psi}+\left(\bar{v}_{2}-\bar{v}_{1}\right) \boldsymbol{v}
\end{array}\right.
$$

where $-k_{2} \leq \bar{v}_{1} \leq k_{2},-\chi \leq \bar{v}_{2} \leq \chi, \boldsymbol{v}=[1,1]^{T}$. It can be seen that $\dot{\boldsymbol{e}}_{\omega} \neq 0$ unless $\boldsymbol{e}_{\psi}=0$ because $k_{1}>k_{2}+\chi$ as per (25). Next, to prove the exponential stability of (23), a candidate Lyapunov function is selected as

$$
W\left(\boldsymbol{e}_{\Psi}, \boldsymbol{e}_{\omega}\right)=c_{1}\left\|\boldsymbol{e}_{\Psi}\right\|^{2}+c_{2} \boldsymbol{e}_{\psi}^{T} \boldsymbol{e}_{\omega}+c_{3}\left\|\boldsymbol{e}_{\omega}\right\|^{2}+c_{4}\left\|\boldsymbol{e}_{\psi}\right\|
$$

where $c_{1}, c_{2}, c_{3}, c_{4}>0$ are constants.

Reformulating (27) as

$$
W\left(\boldsymbol{e}_{\psi}, \boldsymbol{e}_{\omega}\right)=\boldsymbol{\xi}^{T} \boldsymbol{Q} \boldsymbol{\xi}+c_{4}\left\|\boldsymbol{e}_{\psi}\right\|
$$

where

$$
\boldsymbol{\xi}=\left[\boldsymbol{e}_{\Psi}^{T}, \boldsymbol{e}_{\omega}^{T}\right]^{T}, \boldsymbol{Q}=\left[\begin{array}{cc}
c_{1} & c_{2} / 2 \\
c_{2} / 2 & c_{3}
\end{array}\right] .
$$

Apparently, $W\left(\boldsymbol{e}_{\psi}, \boldsymbol{e}_{\omega}\right)$ is positive definite (except for the origin) if $4 c_{1} c_{3}>c_{2}^{2}$. Note that the Lyapunov function is not differentiable at $\boldsymbol{e}_{\psi}=\mathbf{0}$. However, the error dynamics with the condition $\left(\mathbf{0}, \boldsymbol{e}_{\omega}\right)$ can be checked as follows [28]

$$
\left\{\begin{array}{l}
\dot{\boldsymbol{e}}_{\Psi}=\boldsymbol{e}_{\omega} \\
\dot{\boldsymbol{e}}_{\omega}=-k_{2} \frac{\boldsymbol{e}_{\omega}}{\left\|\boldsymbol{e}_{\omega}\right\|}-k_{4} \boldsymbol{e}_{\omega}+\left(\bar{v}_{2}-\bar{v}_{3}\right) \boldsymbol{v}
\end{array}\right.
$$

where $-k_{1} \leq \bar{v}_{3} \leq k_{1}$. It can be seen that $\dot{\boldsymbol{e}}_{\psi} \neq \mathbf{0}$ for nonzero $\boldsymbol{e}_{\omega}$, which means the closed-loop system (23) will not stay on $\boldsymbol{e}_{\psi}=\mathbf{0}$ for $\boldsymbol{e}_{\omega} \neq \mathbf{0}$. Then, taking the derivative of (28) along the system (23) leads to

$$
\begin{aligned}
\dot{W}\left(\boldsymbol{e}_{\Psi}, \boldsymbol{e}_{\omega}\right)= & 2 c_{1} \boldsymbol{e}_{\psi}^{T} \boldsymbol{e}_{\omega}+c_{2}\left\|\boldsymbol{e}_{\omega}\right\|^{2}-k_{1} c_{2}\left\|\boldsymbol{e}_{\psi}\right\|-k_{2} c_{2} \boldsymbol{e}_{\psi}^{T} \boldsymbol{e}_{\omega} /\left\|\boldsymbol{e}_{\omega}\right\| \\
& -k_{3} c_{2}\left\|\boldsymbol{e}_{\Psi}\right\|^{2}-k_{4} c_{2} \boldsymbol{e}_{\Psi}^{T} \boldsymbol{e}_{\omega}-2 k_{1} c_{3} \boldsymbol{e}_{\omega}^{T} \boldsymbol{e}_{\Psi} /\left\|\boldsymbol{e}_{\Psi}\right\| \\
& -2 k_{2} c_{3}\left\|\boldsymbol{e}_{\omega}\right\|-2 k_{3} c_{3} \boldsymbol{e}_{\omega}^{T} \boldsymbol{e}_{\Psi}-2 k_{4} c_{3}\left\|\boldsymbol{e}_{\omega}\right\|^{2} \\
& +c_{4} \boldsymbol{e}_{\Psi}^{T} \boldsymbol{e}_{\omega} /\left\|\boldsymbol{e}_{\Psi}\right\|+c_{2} \boldsymbol{e}_{\Psi}^{T} \boldsymbol{\varepsilon}_{n d i-t w}+2 c_{3} \boldsymbol{e}_{\omega}^{T} \boldsymbol{\varepsilon}_{n d i-t w}
\end{aligned}
$$

To proceed, (29) is rewritten as

$$
\dot{W}\left(\boldsymbol{e}_{\psi}, \boldsymbol{e}_{\omega}\right)=-\left(W_{1}+W_{2}\right)
$$

where

$$
\begin{aligned}
W_{1}= & c_{2} \boldsymbol{e}_{\Psi}^{T}\left(k_{1} \frac{\boldsymbol{e}_{\Psi}}{\left\|\boldsymbol{e}_{\Psi}\right\|}+k_{2} \frac{\boldsymbol{e}_{\omega}}{\left\|\boldsymbol{e}_{\omega}\right\|}-\boldsymbol{\varepsilon}_{n d i-t w}\right)-c_{4} \frac{\boldsymbol{e}_{\Psi}^{T} \boldsymbol{e}_{\omega}}{\left\|\boldsymbol{e}_{\Psi}\right\|} \\
& +2 c_{3} \boldsymbol{e}_{\omega}^{T}\left(k_{2} \frac{\boldsymbol{e}_{\omega}}{\left\|\boldsymbol{e}_{\omega}\right\|}+k_{1} \frac{\boldsymbol{e}_{\Psi}}{\left\|\boldsymbol{e}_{\Psi}\right\|}-\boldsymbol{\varepsilon}_{n d i-t w}\right)
\end{aligned}
$$

$W_{2}=k_{3} c_{2}\left\|\boldsymbol{e}_{\psi}\right\|^{2}+\left(2 k_{4} c_{3}-c_{2}\right)\left\|\boldsymbol{e}_{\psi}\right\|^{2}+\left(2 k_{3} c_{3}+k_{4} c_{2}-2 c_{1}\right) \boldsymbol{e}_{\psi}^{T} \boldsymbol{e}_{\omega}(32)$
Note that $\dot{W}\left(\boldsymbol{e}_{\psi}, \boldsymbol{e}_{\omega}\right)<0$ if $W_{1}>0$ and $W_{2}>0$. From (31), it can be obtained that

$$
\begin{gathered}
W_{1} \geq c_{2}\left\|\boldsymbol{e}_{\Psi}\right\|\left(k_{1}-k_{2}-\chi\right)+2 c_{3}\left\|\boldsymbol{e}_{\omega}\right\|\left(k_{2}-\chi\right) \\
+2 k_{1} c_{3} \boldsymbol{e}_{\omega}^{T} \boldsymbol{e}_{\psi} /\left\|\boldsymbol{e}_{\Psi}\right\|-c_{4} \boldsymbol{e}_{\omega}^{T} \boldsymbol{e}_{\Psi} /\left\|\boldsymbol{e}_{\Psi}\right\|
\end{gathered}
$$

Let $c_{4}$ be selected as $c_{4}=2 k_{1} c_{3} / \bar{m}$ with $\bar{m}>1$, then

$$
W_{1} \geq c_{2}\left\|\boldsymbol{e}_{\Psi}\right\|\left(k_{1}-k_{2}-\chi\right)+2 c_{3}\left\|\boldsymbol{e}_{\omega}\right\|\left(k_{2}-k_{1}-k_{1} / \bar{m}-\chi\right)
$$

It can be seen from (33) that $W_{1}>0$ (except for the origin) if

$$
\left\{\begin{array}{l}
k_{1}>k_{2}+\chi \\
k_{2}>k_{1}(1-1 / \bar{m})+\chi
\end{array}\right.
$$

which can be met by the selection of (25).

For the positivity of $W_{2}, c_{1}, c_{2}, c_{3}$ are chosen such that

$$
\left\{\begin{array}{l}
c_{2}=2 \bar{m} c_{3} \\
2 \bar{m} c_{1}=2 k_{3} c_{3}+\bar{m} k_{4} c_{2}
\end{array}\right.
$$

Therefore, it can be derived that

$$
\frac{c_{2}^{2}}{4 c_{1} c_{3}}=\bar{m}^{2} \frac{c_{3}}{c_{1}}=\frac{\bar{m}^{3}}{k_{3}+k_{4} \bar{m}^{2}}
$$

Obviously, $4 c_{1} c_{3}>c_{2}^{2}$ for the positivity of (28) will not be violated with $k_{4}>\bar{m}$. It can be deduced from (32),(34) that

$$
\begin{aligned}
W_{2}= & 2 \bar{m} k_{3} c_{3}\left\|\boldsymbol{e}_{\psi}\right\|^{2}+\left(2 k_{4} c_{3}-2 \bar{m} c_{3}\right)\left\|\boldsymbol{e}_{\omega}\right\|^{2} \\
& +2 k_{3} c_{3}(1-1 / \bar{m}) \boldsymbol{e}_{\psi}^{T} \boldsymbol{e}_{\omega}=c_{3} \xi^{T} \boldsymbol{R} \xi
\end{aligned}
$$

where

$$
\boldsymbol{R}=\left[\begin{array}{cc}
2 \bar{m} k_{3} & k_{3}(1-1 / \bar{m}) \\
* & 2\left(k_{4}-\bar{m}\right)
\end{array}\right]
$$

Note that $W_{2}>0$ can be ensured if $k_{3}, k_{4}$ are selected such that $\boldsymbol{R}$ is positive definite, which has been implied in (26). From (25),(33),(35), it can be deduced that

$$
\left\{\begin{array}{l}
W_{1} \geq c_{2} \varsigma_{1}\left\|\boldsymbol{e}_{\Psi}\right\| \\
W_{2} \geq c_{3} \lambda_{\min }\{\boldsymbol{R}\}\|\boldsymbol{\xi}\|^{2}
\end{array}\right.
$$

Combining (30) with (36) yields

$$
\begin{aligned}
\dot{W}\left(\boldsymbol{e}_{\Psi}, \boldsymbol{e}_{\omega}\right) & \leq-\left(c_{2} \varsigma_{1}\left\|\boldsymbol{e}_{\Psi}\right\|+c_{3} \lambda_{\min }\{\boldsymbol{R}\}\|\boldsymbol{\xi}\|^{2}\right) \\
& \leq-G_{1}\left(\|\boldsymbol{\xi}\|^{2}+\left\|\boldsymbol{e}_{\Psi}\right\|\right)
\end{aligned}
$$

where $G_{1}=\min \left\{c_{2} s_{1}, c_{3} \lambda_{\min }\{\boldsymbol{R}\}\right\}$. Recall (27) and consider that $\lambda_{\min }\{\boldsymbol{Q}\}\|\boldsymbol{\xi}\|^{2} \leq \boldsymbol{\xi}^{T} \boldsymbol{Q} \boldsymbol{\xi} \leq \lambda_{\max }\{\boldsymbol{Q}\}\|\boldsymbol{\xi}\|^{2}$, it can be derived that

$$
W\left(\boldsymbol{e}_{\Psi}, \boldsymbol{e}_{\omega}\right) \leq \lambda_{\max }\{\boldsymbol{Q}\}\|\xi\|^{2}+c_{4}\left\|\boldsymbol{e}_{\Psi}\right\| \leq G_{2}\left(\|\xi\|^{2}+\left\|\boldsymbol{e}_{\Psi}\right\|\right)
$$

where $G_{2}=\max \left\{\lambda_{\max }\{\boldsymbol{Q}\}, c_{4}\right\}$. Hence, it can be obtained from (37) and (38) that

$$
\dot{W}\left(\boldsymbol{e}_{\psi}, \boldsymbol{e}_{\omega}\right) \leq-\frac{G_{1}}{G_{2}} W\left(\boldsymbol{e}_{\psi}, \boldsymbol{e}_{\omega}\right)
$$

Therefore, the system is globally exponentially stable. In other words, the error dynamics $\boldsymbol{e}_{\psi}$ and $\boldsymbol{e}_{\omega}$ can simultaneously converge to the origin exponentially. This completes the proof.

Remark 3. Although the NDI-TW controller has the property of cancelling perturbations, there exist several conservativeness: i) The strong dependency on the model knowledge (both $\bar{A}$ and $\overline{\boldsymbol{B}}$ are required in (21)); ii) The residual $\boldsymbol{\varepsilon}_{n d i-t w}$ increases when the knowledge of $\overline{\boldsymbol{A}}, \overline{\boldsymbol{B}}$ becomes worse (see (24)); iii) The larger the residual's absolute values, the greater minimum values of the control gains $k_{1}, k_{2}$ are required as per (25). Therefore, it is challenging and contradictory to reduce the model dependency and uncertainty residual simultaneously for the NDI-TW controller. This issue will be mitigated by designing the INDI-TW controller in the next subsection. 


\section{B. INDI-TW Controller Design and Analysis}

This section derives an INDI-TW controller that exploits the incremental control concept [18] in which the angular acceleration measurement and partial model dynamics are used. To begin with, the second equation of (16) is rewritten by considering $\boldsymbol{A}, \boldsymbol{B}$ are functions of the system state (denoted as $\boldsymbol{x})$ and fault trigger $\delta(t)$ as

$$
\dot{\boldsymbol{e}}_{\omega}=\boldsymbol{A}(\boldsymbol{x}, \delta)+\boldsymbol{B}(\boldsymbol{x}, \delta) \boldsymbol{u}-\ddot{\boldsymbol{\Psi}}_{c}+\boldsymbol{\Omega}
$$

Then, the incremental dynamics for $\boldsymbol{e}_{\omega}$ is derived via taking the first-order Taylor series expansion of (39) around the instant $t-\Delta t$ (described by the subscript $0, \Delta t$ is the sampling interval) [18] as follows

$$
\begin{aligned}
\dot{\boldsymbol{e}}_{\omega}= & \dot{\boldsymbol{e}}_{\omega 0}+\left.\frac{\partial[\boldsymbol{A}(\boldsymbol{x}, \delta)+\boldsymbol{B}(\boldsymbol{x}, \delta) \boldsymbol{u}]}{\partial \boldsymbol{x}}\right|_{0} \Delta \boldsymbol{x}+\boldsymbol{B}\left(\boldsymbol{x}_{0}, \delta_{0}\right) \Delta \boldsymbol{u}-\Delta \ddot{\boldsymbol{\Psi}}_{c} \\
& +\left.\frac{\partial[\boldsymbol{A}(\boldsymbol{x}, \delta)+\boldsymbol{B}(\boldsymbol{x}, \delta) \boldsymbol{u}]}{\partial \delta}\right|_{0} \Delta \delta+\Delta \boldsymbol{\Omega}+\mathcal{O}\left(\Delta \boldsymbol{x}^{2}\right)
\end{aligned}
$$

where $\dot{\boldsymbol{e}}_{\omega 0}$ is the estimated/measured derivative of the error dynamics $\boldsymbol{e}_{\omega}$ in the latest step, $\Delta \boldsymbol{x}=\boldsymbol{x}-\boldsymbol{x}_{0}$ and $\Delta \boldsymbol{u}=\boldsymbol{u}-\boldsymbol{u}_{0}$ denote the increments of $\boldsymbol{x}$ and $\boldsymbol{u}$ from $t-\Delta t$ to $t$. $\Delta \delta=\delta-\delta_{0}$ and $\Delta \boldsymbol{\Omega}=\boldsymbol{\Omega}-\boldsymbol{\Omega}_{0}$ depict the fault trigger changes and the variations of the disturbances in $\Delta t$, respectively. $\mathcal{O}\left(\Delta \boldsymbol{x}^{2}\right)$ denotes the higher-order remaining function. Considering (17),(18),(40), then (16) is rewritten as

$$
\left\{\begin{array}{l}
\dot{\boldsymbol{e}}_{\psi}=\boldsymbol{e}_{\omega} \\
\dot{\boldsymbol{e}}_{\omega}=\dot{\boldsymbol{e}}_{\omega 0}+\boldsymbol{B}_{0} \Delta \boldsymbol{u}-\Delta \ddot{\boldsymbol{\Psi}}_{c}+\Delta \boldsymbol{\Omega}+\lambda(\boldsymbol{x}, \delta, \Delta t)
\end{array}\right.
$$

where $\boldsymbol{B}_{0}=\boldsymbol{B}\left(\boldsymbol{x}_{0}, \delta_{0}\right)$, and

$$
\begin{gathered}
\lambda(\boldsymbol{x}, \delta, \Delta t)=\lambda_{1}+\lambda_{2}+\lambda_{3} \\
\lambda_{1}=\frac{\partial[\overline{\boldsymbol{A}}+\Delta \boldsymbol{A}+(\overline{\boldsymbol{B}}+\Delta \boldsymbol{B}) \boldsymbol{u}]}{\partial \boldsymbol{x}} \Delta \boldsymbol{x}+\mathcal{O}\left(\Delta \boldsymbol{x}^{2}\right) \\
\lambda_{2}=\delta_{0} \frac{\partial\left[\left(\boldsymbol{A}_{F}-\overline{\boldsymbol{A}}\right)+\left(\boldsymbol{B}_{F}-\overline{\boldsymbol{B}}\right) \boldsymbol{u}\right]}{\partial \boldsymbol{x}} \Delta \boldsymbol{x} \\
\lambda_{3}=\Delta \delta \underbrace{\left.\left[\left(\boldsymbol{A}_{F}-\overline{\boldsymbol{A}}\right)+\left(\boldsymbol{B}_{F}-\overline{\boldsymbol{B}}\right) \boldsymbol{u}\right]\right|_{0}}_{\lambda_{F}}
\end{gathered}
$$

Assumption 4 [19]. The partial derivatives of $\boldsymbol{A}$ and $\boldsymbol{B}$ with respect to $\boldsymbol{x}$ are bounded for any order.

Assumption 5 [19]. The term $\lambda_{F}$ in (45) is bounded for $\left[t_{F}, t_{F}+\Delta t\right)$.

For the system (41) with relative degree two, the INDI-TW controller is designed as

$$
\Delta \boldsymbol{u}_{\text {indi-tw }}=\overline{\boldsymbol{B}}_{0}^{+}(\underbrace{-\dot{\boldsymbol{e}}_{\omega 0}+\Delta \ddot{\boldsymbol{\Psi}}_{c}}_{\boldsymbol{u}_{e q i}}+\boldsymbol{u}_{t w i})
$$

where $\boldsymbol{u}_{t w i}$ is the same as $\boldsymbol{u}_{t w}$ defined in (21).

Remark 4 . Note that $\dot{\boldsymbol{e}}_{\omega 0}$ can be obtained by angular sensors because $\dot{\boldsymbol{e}}_{\omega}=\boldsymbol{T} \dot{\boldsymbol{\omega}}-\ddot{\boldsymbol{\Psi}}_{c}+\dot{\boldsymbol{T}} \boldsymbol{\omega}$, where $\dot{\boldsymbol{T}} \boldsymbol{\omega}=\overline{\boldsymbol{\Omega}}$ has been included in the lumped disturbance (19) whose effects will be analyzed later. For some cases that $\dot{\omega}$ is not available by sensors, the sampled outputs can be utilized for its estimation [19]. This is not the focus of this work and will not be discussed in detail.
Remark 5. If $k_{1}, k_{2}$ in $\boldsymbol{u}_{t w i}$ are set to zero, then (46) changes from the INDI-TW controller to the INDI controller with reduced robustness.

Remark 6. The unit vectors in $\boldsymbol{u}_{t w}$ and $\boldsymbol{u}_{t w i}$ are modified as $\boldsymbol{e}_{\Psi} /\left(\left\|\boldsymbol{e}_{\psi^{\prime}}\right\|+\varpi\right), \boldsymbol{e}_{\omega} /\left(\left\|\boldsymbol{e}_{\omega}\right\|+\varpi\right)$ to avoid chattering in practical implementation. Increasing $\varpi$ will reduce the system robustness, while reducing $\varpi$ will increase the possibility of chattering, and vice versa.

The whole control command can be presented as

$$
\boldsymbol{u}_{\text {indi-tw }}=\boldsymbol{u}_{0}+\Delta \boldsymbol{u}_{\text {indi-tw }}
$$

where $\boldsymbol{u}_{0}$ is the actuator deflection measured at the latest step $t-\Delta t$. Note that if $\boldsymbol{u}_{0}$ cannot be directly measured, it can be also estimated online [29].

Substituting (46) into (41) leads to

$$
\left\{\begin{array}{l}
\dot{\boldsymbol{e}}_{\Psi}=\boldsymbol{e}_{\omega} \\
\dot{\boldsymbol{e}}_{\omega}=\boldsymbol{u}_{t w i}+\boldsymbol{\varepsilon}_{\text {indi-tw }}
\end{array}\right.
$$

where

$$
\boldsymbol{\varepsilon}_{\text {indi-tw }}=\left(\boldsymbol{B}_{0} \overline{\boldsymbol{B}}_{0}^{+}-\boldsymbol{I}\right)\left(\boldsymbol{u}_{e q i}+\boldsymbol{u}_{t w i}\right)+\Delta \boldsymbol{\Omega}+\lambda(\boldsymbol{x}, \delta, \Delta t)
$$

Then, the following theorem derived from [18] can be obtained.

Theorem 2. Under the Assumptions 1,2,4, and 5, the system residual $\boldsymbol{\varepsilon}_{\text {indi-tw }}$ presented in (48) is ultimately bounded if the condition $\left\|\boldsymbol{I}-\boldsymbol{B}_{0} \overline{\boldsymbol{B}}_{0}^{+}\right\| \leq c<1$ is met for all $t$.

Proof. From (47), the value of $\dot{\boldsymbol{e}}_{\omega}$ for the previous step can be presented as

$$
\dot{\boldsymbol{e}}_{\omega 0}=\boldsymbol{u}_{t w i 0}+\boldsymbol{\varepsilon}_{\text {indi-tw } 0}
$$

Substituting $\boldsymbol{u}_{e q i}=-\dot{\boldsymbol{e}}_{\omega 0}+\Delta \ddot{\boldsymbol{\Psi}}_{c}$ into (48) and combining with (49), it can be derived that

$$
\boldsymbol{\varepsilon}_{\text {indi-tw }}=\left(\boldsymbol{I}-\boldsymbol{B}_{0} \overline{\boldsymbol{B}}_{0}^{+}\right)\left(\varepsilon_{\text {indi-tw } 0}-\Delta \ddot{\boldsymbol{\Psi}}_{c}-\Delta \boldsymbol{u}_{t w i}\right)+\Delta \boldsymbol{\Omega}+\lambda(\boldsymbol{x}, \delta, \Delta t)
$$

where $\Delta \boldsymbol{u}_{t w i}=\boldsymbol{u}_{t w i}-\boldsymbol{u}_{t w i 0}$. For a sufficiently small $\Delta t$, it is assumed that $\Delta \boldsymbol{u}_{t w i}$ is bounded by $\Theta_{1}$ under continuous modification of $\boldsymbol{u}_{t w i}$ in implementation. It can be noted from Assumptions 1 and 2 that $\Delta \ddot{\boldsymbol{\Psi}}_{c}$ and $\Delta \boldsymbol{\Omega}$ are bounded and their bounds are denoted as $\Theta_{2}, \Theta_{3}$, respectively. For $\lambda(x, \delta, \Delta t)$, it can be known that $\lambda_{1}$ and $\lambda_{2}$ are bounded under Assumption 4 because $\|\Delta x\| \rightarrow 0$ with a sufficiently small $\Delta t[19,22]$. Meanwhile, $\lambda_{3}$ is also bounded under Assumption 5 because $\Delta \delta=1$ only for $\left[t_{F}, t_{F}+\Delta t\right)$ and $\Delta \delta=0$ for any other time. Consequently, the boundedness of $\lambda(\boldsymbol{x}, \delta, \Delta t)$ is ensured and denoted as $\Theta_{4}$. Rewriting (50) recursively as

$$
\begin{aligned}
\boldsymbol{\varepsilon}_{\text {indi-tw }}(k)= & \left(\boldsymbol{I}(k)-\boldsymbol{B}_{0}(k) \overline{\boldsymbol{B}}_{0}^{+}(k)\right) \boldsymbol{\varepsilon}_{\text {indi-tw }}(k-1)+\Delta \boldsymbol{\Omega}(k)+\lambda(k) \\
& +\left(\boldsymbol{I}(k)-\boldsymbol{B}_{0}(k) \overline{\boldsymbol{B}}_{0}^{+}(k)\right)\left(-\Delta \ddot{\boldsymbol{\Psi}}_{c}(k)-\Delta \boldsymbol{u}_{t w i}(k)\right)
\end{aligned}
$$

Then, with the condition $\left\|\boldsymbol{I}-\boldsymbol{B}_{0} \overline{\boldsymbol{B}}_{0}^{+}\right\| \leq c<1$, it can be deduced from $[18,19]$ that

$$
\begin{aligned}
\left\|\boldsymbol{\varepsilon}_{\text {indi-tw }}(k)\right\| & \leq c^{k}\left\|\boldsymbol{\varepsilon}_{\text {indi-tw }}(0)\right\|+\left[c\left(\Theta_{1}+\Theta_{2}\right)+\left(\Theta_{3}+\Theta_{4}\right)\right] \sum_{i=1}^{k} c^{k-i} \\
& \leq c^{k}\left\|\boldsymbol{\varepsilon}_{\text {indi-tw }}(0)\right\|+\left[c\left(\Theta_{1}+\Theta_{2}\right)+\left(\Theta_{3}+\Theta_{4}\right)\right] \frac{1-c^{k}}{1-c}
\end{aligned}
$$

Since $c^{k} \rightarrow 0$ as $k \rightarrow+\infty$ for $0 \leq c<1$, and $\left\|\boldsymbol{\varepsilon}_{\text {indi-tw }}(0)\right\|$ is finite in practice, then (51) becomes

$$
\left\|\varepsilon_{\text {indi-tw }}\right\| \leq \bar{\chi}=\frac{c\left(\Theta_{1}+\Theta_{2}\right)+\left(\Theta_{3}+\Theta_{4}\right)}{1-c}
$$

This completes the proof. 
Remark 7. The bound of the residual $\varepsilon_{\text {indi-tw }}$ is related to $\Delta t$, and its boundary can be further reduced with higher sampling frequency $[18,19,22]$.

Remark 8. The condition $\left\|\boldsymbol{I}-\boldsymbol{B}_{0} \overline{\boldsymbol{B}}_{0}^{+}\right\| \leq c<1$ requires $\boldsymbol{B}_{0} \overline{\boldsymbol{B}}_{0}^{+}$to be diagonally dominant, which is commonly used in flight control [18]. Note that it is a condition relaxing Assumption 3. For some cases where the condition is invalid, Assumption 3 should be also made for $\boldsymbol{\varepsilon}_{\text {indi-tw }}$ to ensure the system stability. Particularly, $\left\|\boldsymbol{I}-\boldsymbol{B}_{0} \overline{\boldsymbol{B}}_{0}^{+}\right\|=0$ if $\overline{\boldsymbol{B}}_{0}$ ideally estimates $\boldsymbol{B}_{0}$. It gives a future research direction in the accurate identification of $\boldsymbol{B}_{0}$ such that $\left\|\varepsilon_{\text {indi-tw }}\right\|$ can quickly decay with faster convergence for $c^{k} \rightarrow 0$.

Corollary 1. For the attitude error dynamics (16) (or the transformed incremental dynamics (41)) under uncertainties, faults, and disturbances (17)-(19), the proposed INDI-TW controller (46) can guarantee the simultaneous exponential convergence of $\boldsymbol{e}_{\psi}$ and $\boldsymbol{e}_{\omega}$ under the bounded $\left\|\boldsymbol{\varepsilon}_{\text {indi-tw }}\right\|$ if the control gains $k_{1}, k_{2}$ satisfy

$$
\left\{\begin{array}{l}
k_{1}=k_{2}+\bar{\chi}+\varsigma_{1} \\
k_{2}=(2 \bar{m}-1) \bar{\chi}+(\bar{m}-1) \varsigma_{1}+\varsigma_{2}
\end{array}\right.
$$

and $k_{3}, k_{4}$ satisfy (26).

The dynamics (41) under the INDI-TW controller (46) is presented in (47). Since the condition $\left\|\varepsilon_{\text {indi-tw }}\right\| \leq \bar{\chi}$ can be guaranteed/assumed when $\left\|\boldsymbol{I}-\boldsymbol{B}_{0} \overline{\boldsymbol{B}}_{0}^{+}\right\| \leq c<1$ is met or not, the proof for Corollary 1 is similar to that for Theorem 1 and is not repeated here for space limit.

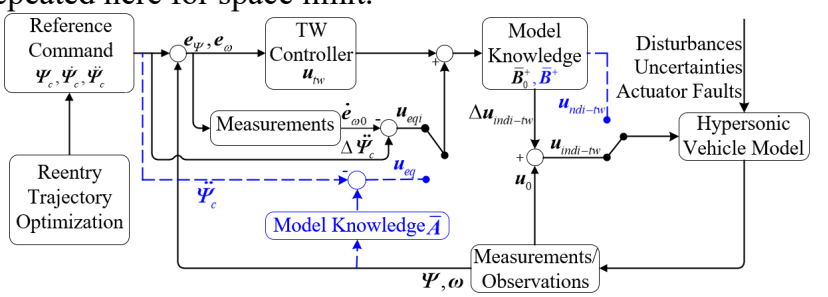

Fig. 1. Control structure for INDI-TW and NDI-TW

The NDI-TW and INDI-TW control structure for the hypersonic vehicle attitude tracking can be presented in Fig. 1. Therein, the INDI-TW or NDI-TW controller is used when the two switches are connected to the black solid lines or blue dashed lines, respectively. It can be seen that the same twisting controller is used, but different equivalent controllers are adopted with/without the model knowledge of $\overline{\boldsymbol{A}}$.

Remark 9. The conservativeness in the NDI-TW controller has been solved by the INDI-TW controller in the following aspects: $i$ ) The dependency on the model knowledge is reduced ( $\overline{\boldsymbol{A}}$ is not required but needed in NDI-TW); ii) The uncertainty residual is less dependent on the model knowledge; iii) Thanks to the relaxed dependency and incremental nature, the INDI-TW can reduce the magnitude of $\varepsilon_{\text {indi-tw }}$, compared with $\boldsymbol{\varepsilon}_{n d i-t w}$ in NDI-TW. This implies that smaller minimum values of $k_{1}, k_{2}$ can be attained in INDI-TW than NDI-TW. The reduction becomes more significant as the uncertainty residual on $\boldsymbol{A}$ becomes bigger or $\lambda$ becomes smaller.

\section{Comparisons Between NDI-TW and INDI-TW}

i) Hypothesis and Model Dependency: As a kind of the INDI-SMC controllers [18], INDI-TW relaxes Assumption 3 in
Theorem 2. The INDI-TW does not require $\overline{\boldsymbol{A}}$, which is a key to compensate for the real dynamics in $\boldsymbol{A}$ in NDI-TW.

ii) Robustness: The robustness of the INDI-TW controller is better than the NDI-TW controller because 1) The deviation between the dynamics in $\boldsymbol{A}$ and $\overline{\boldsymbol{A}}$ is eliminated in INDI-TW because it circumvents the estimation of $\boldsymbol{A} ; 2$ ) The side effects incurred by uncertain and faulty dynamics are compensated in INDI-TW by the latest measurement $\dot{\boldsymbol{e}}_{\omega 0}$ and $\boldsymbol{u}_{0}$.

iii) Fault Tolerance: Considering the uncertain and faulty dynamics (17),(18), the residuals (24) and (48) are rewritten as

$$
\begin{gathered}
\boldsymbol{\varepsilon}_{n d i-t w}=\left(\boldsymbol{A}_{F}-\overline{\boldsymbol{A}}\right) \delta(t)+\Delta \boldsymbol{A}+\left[\left(\boldsymbol{B}_{F}-\overline{\boldsymbol{B}}\right) \delta(t)+\Delta \boldsymbol{B}\right] \boldsymbol{u}_{n d i-t w}+\boldsymbol{\Omega} \\
\boldsymbol{\varepsilon}_{\text {indi-tw }}=\left.\left[\left(\boldsymbol{B}_{F}-\overline{\boldsymbol{B}}\right) \delta(t)+\Delta \boldsymbol{B}\right]\right|_{0} \Delta \boldsymbol{u}_{\text {indi-tw }}+\Delta \boldsymbol{\Omega}+\lambda(\boldsymbol{x}, \delta, \Delta t)
\end{gathered}
$$

Then, it can be seen from (42),(44),(45),(53),(54) that $\boldsymbol{\varepsilon}_{\text {ndi-tw }}$ will be affected by $\boldsymbol{A}_{F}-\overline{\boldsymbol{A}}$ more heavily than $\boldsymbol{\varepsilon}_{\text {indi-tw }}$ under the faulty case. Moreover, the fault influences on $\boldsymbol{\varepsilon}_{\text {indi-tw }}$ can be further reduced with smaller $\Delta t$.

iv) Residual Magnitude: Based on (20),(42) (45), the upper bounds of (53) and (54) can be presented as

$$
\begin{aligned}
& \int\left\|\boldsymbol{\varepsilon}_{n d i-t w}\left(t<t_{F}\right)\right\|=\left\|\Delta \boldsymbol{A}+\Delta \boldsymbol{B} \boldsymbol{u}_{n d i-t w}+\boldsymbol{\Omega}\right\| \leq\|\Delta \boldsymbol{A}\|+\|\Delta \boldsymbol{B}\|\left\|\boldsymbol{u}_{n d i-t w}\right\|+\|\boldsymbol{\Omega}\| \\
& \left\{\left\|\varepsilon_{\text {indi-tw }}\left(t<t_{F}\right)\right\|=\left\|\Delta \boldsymbol{B}_{0} \Delta \boldsymbol{u}_{\text {indi-tw }}+\Delta \boldsymbol{\Omega}+\lambda_{1}\right\| \leq\left\|\Delta \boldsymbol{B}_{0}\right\|\left\|\Delta \boldsymbol{u}_{\text {indi-tw }}\right\|+\|\Delta \boldsymbol{\Omega}\|+\left\|\lambda_{1}\right\|\right. \\
& \int\left\|\boldsymbol{\varepsilon}_{n d i-t w}\left(t \geq t_{F}\right)\right\|=\left\|\boldsymbol{A}_{F}-\overline{\boldsymbol{A}}+\Delta \boldsymbol{A}+\left[\left(\boldsymbol{B}_{F}-\overline{\boldsymbol{B}}\right)+\Delta \boldsymbol{B}\right] \boldsymbol{u}_{n d i-t w}+\boldsymbol{\Omega}\right\| \\
& \leq\left\|\boldsymbol{A}_{F}-\overline{\boldsymbol{A}}+\Delta \boldsymbol{A}\right\|+\|\Delta \boldsymbol{B}\|\left\|\boldsymbol{u}_{n d i-t w}\right\|+\left\|\boldsymbol{B}_{F}-\overline{\boldsymbol{B}}\right\|\left\|\boldsymbol{u}_{\text {ndi-tw }}\right\|+\|\boldsymbol{\Omega}\| \\
& \left\{\left\|\boldsymbol{\varepsilon}_{\text {indi-tw }}\left(t \geq t_{F+}\right)\right\|=\left\|\left.\left[\left(\boldsymbol{B}_{F}-\overline{\boldsymbol{B}}\right)+\Delta \boldsymbol{B}\right]\right|_{0} \Delta \boldsymbol{u}_{\text {indi-tw }}+\Delta \boldsymbol{\Omega}+\lambda_{1}+\lambda_{2}\right\|\right. \\
& \leq\left\|\boldsymbol{B}_{F}-\overline{\boldsymbol{B}}\right\|\left\|_{0}\right\| \Delta \boldsymbol{u}_{\text {indi-tw }}\|+\| \Delta \boldsymbol{B}\left\|_{0}\right\| \Delta \boldsymbol{u}_{\text {indi-tw }}\|+\| \Delta \boldsymbol{\Omega}\|+\| \lambda_{1}+\lambda_{2} \| \\
& \left\|\boldsymbol{\varepsilon}_{\text {indi-tw }}\left(t_{F} \leq t<t_{F+}\right)\right\|=\left\|\left.\left[\left(\boldsymbol{B}_{F}-\overline{\boldsymbol{B}}\right)+\Delta \boldsymbol{B}\right]\right|_{0} \Delta \boldsymbol{u}_{\text {indi-tw }}+\Delta \boldsymbol{\Omega}+\lambda\right\| \\
& \leq\left\|\boldsymbol{B}_{F}-\overline{\boldsymbol{B}}\right\|\left\|_{0}\right\| \Delta \boldsymbol{u}_{\text {indi-tw }}\|+\| \Delta \boldsymbol{B}\left\|_{0}\right\| \Delta \boldsymbol{u}_{\text {indi-tw }}\|+\| \Delta \boldsymbol{\Omega}\|+\| \lambda_{1}+\lambda_{2} \| \\
& +\left\|\boldsymbol{A}_{F}-\overline{\boldsymbol{A}}\right\|_{0}+\left\|\boldsymbol{B}_{F}-\overline{\boldsymbol{B}}\right\|_{0}\left\|\boldsymbol{u}_{\text {indi-tw }}\right\|
\end{aligned}
$$

where $t_{F+}=t_{F}+\Delta t$. As per (43),(44), $\left\|\lambda_{1}\right\|$ and $\left\|\lambda_{1}+\lambda_{2}\right\|$ are negligible for a small $\Delta t$. Meanwhile, if $\boldsymbol{\Omega} \neq 0$ and $\boldsymbol{u}_{n d i-t w} \neq 0$, there exists a sufficiently small $\Delta t$ such that $\|\Delta \boldsymbol{\Omega}\|<\|\boldsymbol{\Omega}\|$, $\left\|\Delta \boldsymbol{u}_{\text {indi-tw }}\right\|<\left\|\boldsymbol{u}_{n d i-t w}\right\|[18,19]$ under continuous modification of $\boldsymbol{u}_{t w}$ and $\boldsymbol{u}_{t w i}$ in implementation. Hence, a smaller bound of $\boldsymbol{\varepsilon}_{\text {indi-tw }}$ can be attained for a small $\Delta t$ than $\boldsymbol{\varepsilon}_{n d i-t w}$ except for $t \in\left[t_{F}, t_{F+}\right)$, during which $\varepsilon_{\text {indi-tw }}$ and $\boldsymbol{\varepsilon}_{n d i-t w}$ are comparable. As for the guidance of choosing $\Delta t$, one can refer to [30].

Remark 10. The gains $k_{1}, k_{2}$ for both NDI-TW and INDI-TW controllers are related to $\chi$ and $\bar{\chi}$, respectively. It is alternative to use gain adaption methods to update $k_{1}, k_{2}$ such as the adaptive twisting controller in [24]. However, it is not the focus in this work and does not affect the comparative analysis for $\boldsymbol{\varepsilon}_{\text {indi-tw }}$ and $\boldsymbol{\varepsilon}_{n d i-t w}$.

To sum up, the INDI-TW-based FTC for hypersonic vehicles has the following theoretical and practical significance: $i$ ) The dependency on part of the model knowledge (dynamics in $\boldsymbol{A}$ ) is removed for controller design and uncertainty induction; $i$ ) Small and fixed gains can be attained for full trajectory tracking due to reduced residual magnitude, which improves robustness and attenuates chattering; iii) Fault tolerance and tracking accuracy can be enhanced with higher sampling frequency. 
IEEE Transactions on Industrial Informatics

TABLE I

INITIAL FLIGHT CONDITIONS OF THE HYPERSONIC VEHICLE

\begin{tabular}{cccc}
\hline \hline Variables & Values & Variables & Values \\
\hline Hight & $60 \mathrm{~km}$ & AOA & $5 \mathrm{deg}$ \\
Speed & $4000 \mathrm{~m} / \mathrm{s}$ & Sideslip Angle & $5 \mathrm{deg}$ \\
Flight Path Angle & $0 \mathrm{deg}$ & Bank Angle & $-13.4 \mathrm{deg}$ \\
Longitude & $45 \mathrm{deg}$ & Pitch Rate & $1 \mathrm{deg} / \mathrm{s}$ \\
Latitude & $30 \mathrm{deg}$ & Roll Rate & $1 \mathrm{deg} / \mathrm{s}$ \\
Heading Angle & $0 \mathrm{deg}$ & Yaw Rate & $1 \mathrm{deg} / \mathrm{s}$ \\
\hline \hline
\end{tabular}

\section{NUMERICAL VERIFICATION}

The performance of the NDI-TW and INDI-TW is validated for the tracking of a hypersonic vehicle's reentry trajectory. The vehicle model in [26] is adopted. Specifically, the mass, reference area, reference length, and the moments of inertia are $1200 \mathrm{~kg}, 0.446 \mathrm{~m}^{2}, 0.98 \mathrm{~m}, I_{x x}=100 \mathrm{~kg} \cdot \mathrm{m}^{2}, I_{y y}=5600 \mathrm{~kg} \cdot \mathrm{m}^{2}$, $I_{z z}=5700 \mathrm{~kg} \cdot \mathrm{m}^{2}$, respectively. The aerodynamic coefficients in [26] are used for the trajectory design and attitude tracking. The initial conditions are shown in Table I. The attitude commands are obtained by optimizing the reentry trajectory via the Gauss Pseudo-Spectral Method to meet the following constraints:

$h_{f}=30 \mathrm{~km}, V_{f}=2300 \mathrm{~m} / \mathrm{s}, \gamma_{f}=-1.9^{\circ}, \theta_{f}=55^{\circ},\left[\alpha_{\min }, \alpha_{\max }\right]=\left[5^{\circ}, 15^{\circ}\right]$,

$\left[\sigma_{\min }, \sigma_{\max }\right]=\left[-60^{\circ}, 60^{\circ}\right], \bar{q} \leq 120 \mathrm{kpa}, n \leq 5 \mathrm{~g}, \dot{\Pi} \leq 1300 \mathrm{KW} \cdot \mathrm{m}^{2}$,

where $n$ is the total overload, $\dot{\Pi}$ is the heating flux density, the subscript " $\mathrm{f}$ " corresponds to final states. The objective is to meet the minimum flight time, which is $297.24 \mathrm{~s}$. The control gains for NDI-TW are selected by the following steps:

i) $\bar{m}=1.1, k_{3}=2, k_{4}=2$ are selected to meet $\bar{m}>1, k_{3}>0$,

$k_{4}>\bar{m}$, and the condition in (26);

ii) $\varsigma_{1}=\varsigma_{2}=0.01$ are chosen to satisfy $\varsigma_{1}>0, \varsigma_{2}>0$;

iii) $k_{1} \geq 0.68$ and $k_{2} \geq 0.37$ are calculated from (25) by estimating a conservative $\chi=0.3$.

Then, $k_{1}=0.8$ and $k_{2}=0.7$ can be easily tuned to meet acceptable dynamic responses. The control gains for INDI-TW are selected the same as NDI-TW for a fair comparison. The INDI controller with $\left[k_{1}, k_{2}, k_{3}, k_{4}\right]=[0,0,2.2,2]$ is also verified. The nominal $\overline{\boldsymbol{A}}$ and/or $\overline{\boldsymbol{B}}$ are used for all controllers. The sampling frequency is $200 \mathrm{HZ} . \varpi=0.1$ is selected to make a balance between chattering issue and system robustness according to Remark 6 . The actuator dynamics is modeled by a second-order transfer function [25] with the natural frequency of $20 \mathrm{HZ}$ and the damping ratio of 0.7 . Besides, the actuator's position and rate are limited by $|\delta| \leq 20^{\circ}$ and $|\dot{\delta}| \leq 100 \% \mathrm{~s}$.
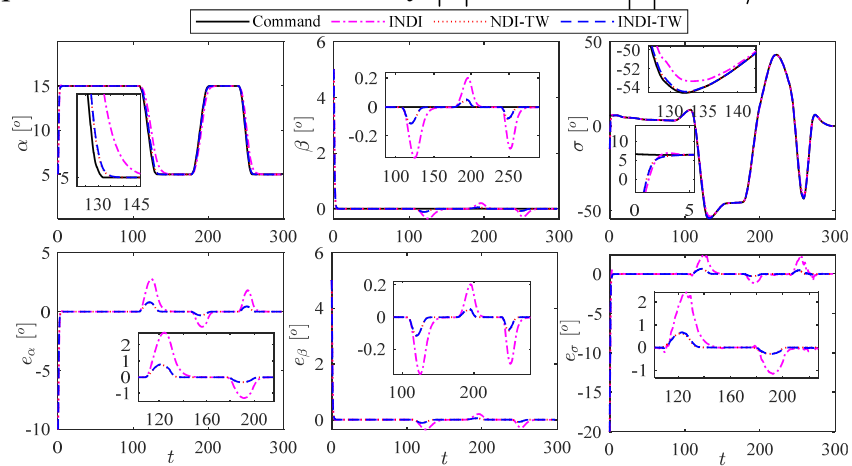

Fig. 2 Attitude responses and tracking errors under the nominal condition

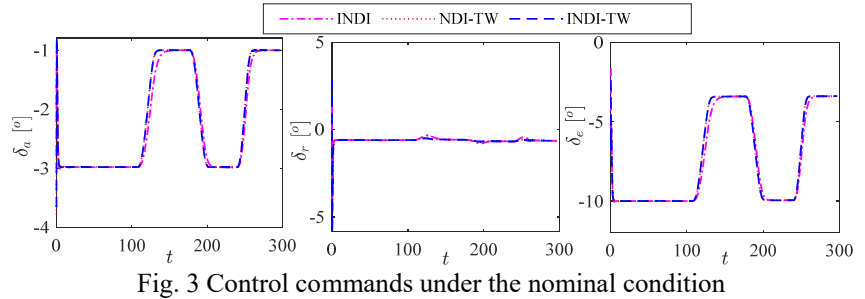

\section{A. Verifications Under Nominal Condition}

The first set of simulations is conducted under the nominal condition. As can be seen from Fig. 2, all the controllers can precisely track the attitude commands during the whole reentry phase. Only small errors arise during the AOA transition intervals. Both NDI-TW and INDI-TW have almost the same performance, while INDI creates slightly larger errors because it's less robust against the strong nonlinearities and couplings in real flight dynamics. Fig. 3 shows that smooth and normal control commands are generated by all controllers. Moreover, it can be observed from Fig. 4 that the vehicle tracks the reference trajectory precisely with small deviations.

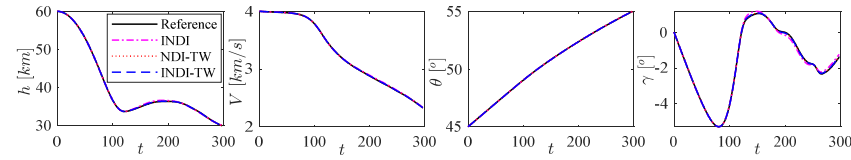

Fig. 4 Flight trajectory responses under the nominal condition

\section{B. Verifications Under External Disturbances}

To evaluate the robustness of each controller, the external disturbances are chosen as $\boldsymbol{d}=\bar{d} \times[1+\sin (\pi t / 100)+\sin (\pi t / 200)$, $1+\sin (\pi t / 100)+\cos (\pi t / 200), 1+\cos (\pi t / 100)+\sin (\pi t / 200)]^{T} . \bar{d}$ is the magnitude to be tested. It can be seen from Fig. 5 that the tracking performance is rarely influenced compared to Fig. 2 when $\bar{d}$ is set to 0.01 . However, the NDI-TW and INDI create larger tracking errors than INDI-TW when $\bar{d}$ increases to 0.1 , as shown in Fig. 6. Note that the INDI and INDI-TW can still ensure stable tracking in this case, while the NDI-TW cannot.

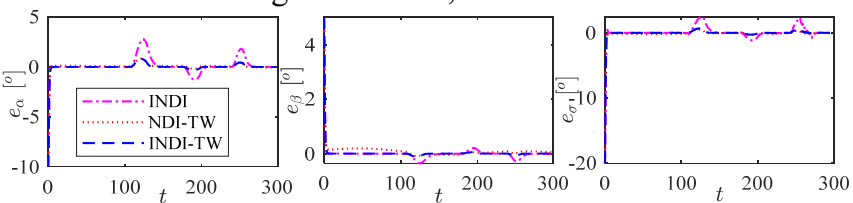

Fig. 5 Attitude tracking errors under external disturbances with $\bar{d}=0.01$



Fig. 6 Attitude tracking errors under external disturbances with $\bar{d}=0.1$

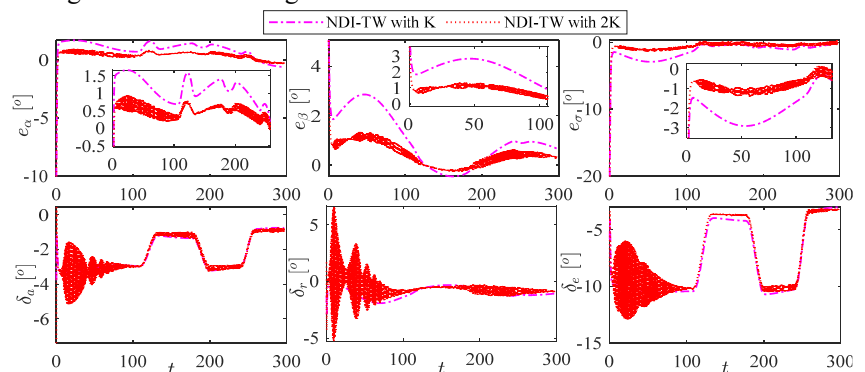

Fig. 7 NDI-TW with different gains under external disturbances with $\bar{d}=0.1$ 
It is worth noting that the tracking errors can be reduced by NDI-TW if higher control gains are set. To this end, the attitude tracking errors and control commands under NDI-TW with $\mathrm{K}$ and $2 \mathrm{~K}$ ( $\mathrm{K}$ denotes all the control gains) are compared in Fig. 7. It can be observed that the tracking errors are reduced by high gains, but the chattering arises, which is undesirable in practice.

\section{Verifications Under Disturbances and Actuator Faults}

The external disturbances with $\bar{d}=0.1$ and actuator faults are simultaneously considered to validate the fault-tolerance and robustness of the three controllers. Four actuators are considered as $\boldsymbol{u}=\left[\delta_{l e}, \delta_{u r}, \delta_{r e}, \delta_{l r}\right]^{T}$ whose components are left elevon, upper rudder, right elevon, and lower rudder, respectively. The allocation matrix satisfying $\boldsymbol{C u}=\left[\delta_{a}, \delta_{r}, \delta_{e}\right]^{T}$ is $\boldsymbol{C}=[0.25,0.25,-0.25,-0.25 ; 0,0.5,0,0.5 ; 0.5,0,0.5,0]^{T}$. The left elevon $\delta_{l e}$ suddenly lost its effectiveness by $50 \%$ during the reentry phase at $t=10 \mathrm{~s}$. This fault will cause severe perturbations in the roll and pitch moments. Therefore, the tracking of AOA and bank angle is disturbed, and thus the sideslip angle will be affected in turn due to the strong couplings among them.

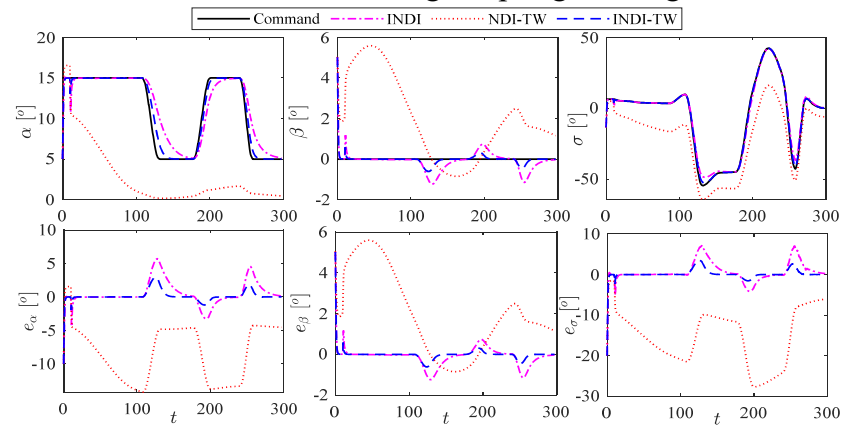

Fig. 8 Attitude responses and errors under disturbances and actuator faults

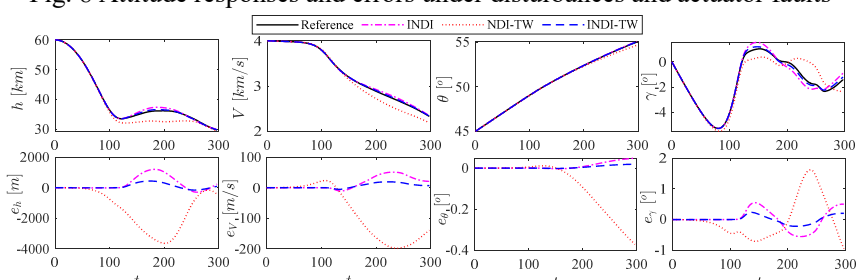

Fig. 9 Trajectory responses and errors under disturbances and actuator faults

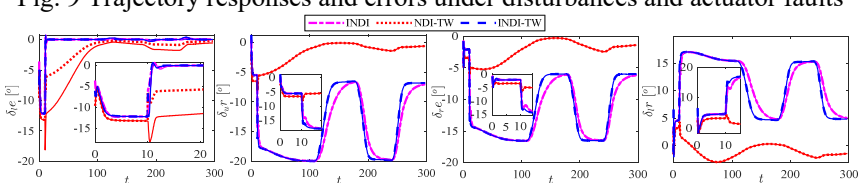

Fig. 10 Fin deflections under external disturbances and actuator faults

From Figs. 8 and 9, it can be noted that the NDI-TW is incapable of handling the fault and the states leave away from the references with large errors. The other two controllers succeed in the tracking mission, and the INDI-TW has better performance and smaller errors than INDI. The attitudes controlled by INDI and INDI-TW diverge first after the fault occurs but are quickly driven back to their stable points. This is because the fault case is recovered using the remaining healthy actuators, as can be seen in Fig. 10. It can be observed that $\delta_{l e}$ under INDI and INDI-TW is near zero after fault happens such that the vehicle will depend less on the left elevon. At the same time, the other three actuators make immediate responses to the faulty actuator for recovery. As shown in Fig. 11, the norm of the uncertainty residual by NDI-TW is greater than that by INDI-TW, which validates the analysis in Sec. III.C.

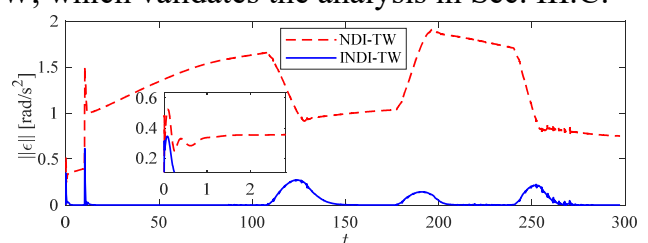

Fig. 11 The norm of uncertainty residuals by NDI-TW and INDI-TW

For the sensor-based controller investigated in this paper, the control performance under noisy measurements is further demonstrated using the INDI-TW controller. Specifically, Gaussian measurement noise with zero mean and standard deviations of $0.001 \mathrm{rad} / \mathrm{s}, 0.01 \mathrm{rad} / \mathrm{s}^{2}$, and $0.01 \mathrm{rad}$ is considered for the angular rate ( $\omega$ ), the latest angular acceleration-related $\left(\dot{\boldsymbol{e}}_{\omega 0}\right)$ term, and the latest actuator $\left(\boldsymbol{u}_{0}\right)$, respectively. The simulation setting is the same as that described in the beginning of this subsection. In addition, a low-pass filter is employed to smooth the noisy measurement. The attitude and actuator responses are shown in Figs. 12 and 13, respectively. It can be seen that the tracking errors are acceptable, and the tracking performance as well as the fin deflections are not affected much, compared to the results under INDI-TW in Figs. 8 and 10.

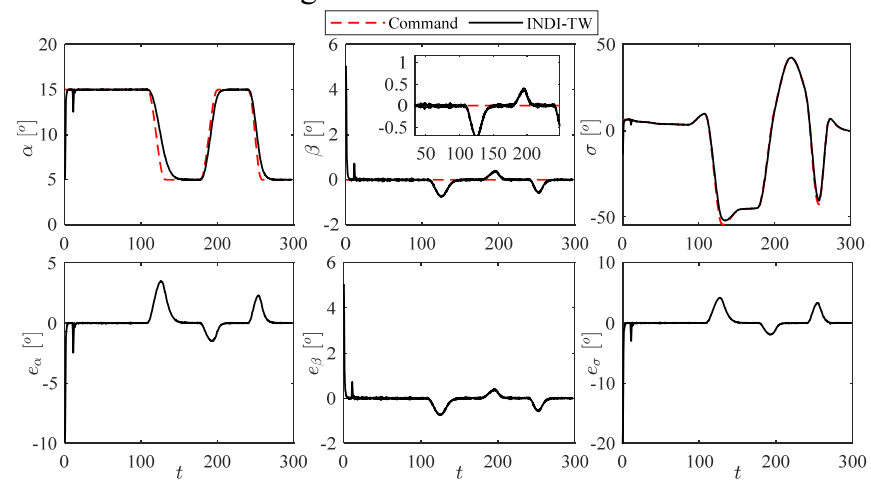

Fig. 12 Attitude responses and errors considering measurement noise

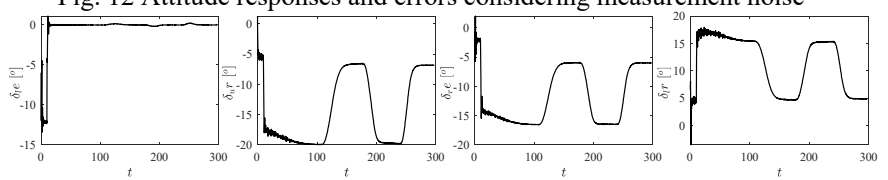

Fig. 13 Fin deflections considering measurement noise

\section{CONCLUSION}

The full reentry trajectory tracking problem for hypersonic vehicles is solved considering modelling uncertainties, external disturbances, and actuator faults. A multivariable twisting controller is proposed to ensure the tracking error and its first-order derivative converge to the origin simultaneously and exponentially. To depend less on the model knowledge, an incremental twisting fault tolerant controller is developed and its closed-loop stability is proved with theoretical guarantees on the boundedness of uncertainty residuals. Simulations validate that both the INDI-TW and INDI can feasibly track the full trajectory with fixed control gains even under disturbances, uncertainties, and actuator faults, while NDI-TW fails the same mission. Comparison studies show that the INDI-TW has better 
robustness and fault tolerance compared to the NDI-TW and INDI. Although the tracking errors can be reduced by NDI-TW with high gains, undesirable chattering is introduced. The future research will focus on the input saturation and estimation of angular acceleration.

\section{REFERENCES}

[1] J. Sun, Z. Pu, J. Yi, and Z. Liu, "Fixed-time control with uncertainty and measurement noise suppression for hypersonic vehicles via augmented sliding mode observers," IEEE Transactions on Industrial Informatics, vol. 16, no. 2, pp. 1192-1203, Oct. 2019.

[2] H. An, J. Liu, C. Wang, and L. Wu, "Approximate back-stepping fault-tolerant control of the flexible air-breathing hypersonic vehicle," IEEE/ASME Transactions on Mechatronics, vol. 21, no. 3, pp. 1680-1691, Dec. 2015

[3] Z. Wang, B. Liang, Y. Sun, and T. Zhang, "Adaptive fault-tolerant prescribed-time control for teleoperation systems with position error constraints," IEEE Transactions on Industrial Informatics, vol. 16, no. 7, pp. 4889-4899, Jul. 2020.

[4] B. Guo, and Y. Chen, "Robust adaptive fault-tolerant control of four-wheel independently actuated electric vehicles," IEEE Transactions on Industrial Informatics, vol. 16, no. 5, pp. 2882-2894, May. 2020.

[5] B. Xian, and W. Hao, "Nonlinear robust fault-tolerant control of the tilt trirotor UAV under rear servo's stuck fault: theory and experiments," IEEE Transactions on Industrial Informatics, vol. 15, no. 4, pp. 2158-2166, Apr. 2019

[6] Q. Hu, X. Shao, and W. H. Chen, "Robust fault-tolerant tracking control for spacecraft proximity operations using time-varying sliding mode," IEEE Transactions on Aerospace and Electronic Systems, vol. 54, no. 1, pp. 2-17, Jul. 2017.

[7] Y. Zhang, and J. Jiang, "Bibliographical review on reconfigurable fault-tolerant control systems," Annual reviews in control, vol. 32, no. 2 , pp. 229-252, Dec. 2008.

[8] Q. Shen, B. Jiang, and V. Cocquempot, "Fault-tolerant control for T-S fuzzy systems with application to near-space hypersonic vehicle with actuator faults," IEEE Transactions on Fuzzy Systems, vol. 20, no. 4, pp. 652-665, Dec. 2011

[9] A. Abbaspour, K. K. Yen, P. Forouzannezhad, and A. Sargolzaei, "A neural adaptive approach for active fault-tolerant control design in UAV," IEEE Transactions on Systems, Man, and Cybernetics: Systems, vol. 50, no. 9 , pp. 3401-3411, Jul. 2018

[10] K. Rudin, G. J. Ducard, and R. Y. Siegwart, "Active fault tolerant control with imperfect fault detection information: applications to UAVs," IEEE Transactions on Aerospace and Electronic Systems, vol. 56, no. 4, pp. 2792-2805, Dec. 2019

[11] Q. Shen, C. Yue, C. H. Goh, and D. Wang, "Active fault-tolerant control system design for spacecraft attitude maneuvers with actuator saturation and faults," IEEE Transactions on Industrial Electronics, vol. 66, no. 5, pp. 3763-3772, May. 2019.

[12] J. G. Sun, S. M. Song, and G. Q. Wu, "Fault-tolerant track control of hypersonic vehicle based on fast terminal sliding mode," Journal of Spacecraft and Rockets, vol. 54, no. 6, pp. 1304-1316, Sep. 2017

[13] X. Yu, P. Li, and Y. Zhang, "The design of fixed-time observer and finite-time fault-tolerant control for hypersonic gliding vehicles," IEEE Transactions on Industrial Electronics, vol. 65, no. 5, pp. 4135-4144, Nov. 2017.

[14] Y. Yuan, Z. Wang, L. Guo, and H. Liu, "Barrier Lyapunov functions-based adaptive fault tolerant control for flexible hypersonic flight vehicles with full state constraints," IEEE Transactions on Systems, Man, and Cybernetics: Systems, vol. 50, no. 9, pp. 3391-3400, Jul. 2018.

[15] W. Ren, B. Jiang, and H. Yang, "Singular perturbation-based fault-tolerant control of the air-breathing hypersonic vehicle," IEEE/ASME Transactions on Mechatronics, vol. 24, no. 6, pp. 2562-2571, Oct. 2019.

[16] S. Sieberling, Q. P. Chu, and J. A. Mulder, "Robust flight control using incremental nonlinear dynamic inversion and angular acceleration prediction," Journal of Guidance, Control, and Dynamics, vol. 33, no. 6 , pp. 1732-1742, 2010

[17] D. I. Ignatyev, H. S. Shin, A. Tsourdos, "Two-layer adaptive augmentation for incremental backstepping flight control of transport aircraft in uncertain conditions," Aerospace Science and Technology, vol. 105, p. 106051 , Oct. 2020
[18] X. Wang, E. J. V. Kampen, Q. Chu, and P. Lu, "Incremental sliding-mode fault-tolerant flight control," Journal of guidance, control, and dynamics, vol. 42, no. 2, pp. 244-259, Feb. 2019.

[19] X. Wang, S. Sun, E. J. V. Kampen, and Q. Chu, "Quadrotor fault tolerant incremental sliding mode control driven by sliding mode disturbance observers," Aerospace Science and Technology, vol. 87, pp. 417-430, Apr. 2019.

[20] E. J. J. Smeur, Q. Chu, and G. C. H. E. D. Croon, "Adaptive incremental nonlinear dynamic inversion for attitude control of micro air vehicles," Journal of Guidance, Control, and Dynamics, vol. 39, no. 3, pp. 450-461, Mar. 2016.

[21] S. Sun, X. Wang, Q. Chu, and C. D. Visser, "Incremental nonlinear fault-tolerant control of a quadrotor with complete loss of two opposing rotors," IEEE Transactions on Robotics, Early Access, Aug. 2020.

[22] X. Wang, E. J. V. Kampen, and Q. Chu, "Quadrotor fault-tolerant incremental nonsingular terminal sliding mode control," Aerospace Science and Technology, vol. 95, p. 105514, Dec. 2019.

[23] A. Levant, "Sliding order and sliding accuracy in sliding mode control," International journal of control, vol. 58, no. 6, pp. 1247-1263, Dec. 1993.

[24] Z. Guo, J. Chang, J. Guo, and J. Zhou, "Adaptive twisting sliding mode algorithm for hypersonic reentry vehicle attitude control based on finite-time observer," ISA transactions, vol. 77, pp. 20-29, Jun. 2018.

[25] S. Mathavaraj, O. Halbe, and R. Padhi, "Robust control of a reusable launch vehicle in reentry phase using model following neuro-adaptive design," In AIAA guidance, navigation, and control conference, p. 8312, Toronto, Ontario Canada, Aug. 2010.

[26] J. Wang, L. Liu, T. Zhao, and G. Tang, "Integrated guidance and control for hypersonic vehicles in dive phase with multiple constraints," Aerospace Science and Technology, vol. 53, pp. 103-115, Jun. 2016.

[27] Z. Guo, J. Guo, J. Zhou, and J. Chang, "Robust tracking for hypersonic reentry vehicles via disturbance estimation-triggered control", IEEE Transactions on Aerospace and Electronic Systems, vol. 56, no. 2, pp. 1279-1289, Apr. 2020.

[28] C. A. M. Fuentes, R. Seeber, L. Fridman, and J. A. Moreno, "Saturated Lipschitz continuous sliding mode controller for perturbed systems with uncertain control coefficient," IEEE Transactions on Automatic Control, Early Access, Oct. 2020.

[29] P. Acquatella, E. V. Kampen, Q. Chu, "Incremental backstepping for robust nonlinear flight control," In CEAS Conference on Guidance, Navigation, and Control, pp. 1444-1463, Delft, The Netherlands, Apr. 2013

[30] X. Wang, E. J. V. Kampen, Q. Chu, and P. Lu, "Stability analysis for incremental nonlinear dynamic inversion control," Journal of Guidance, Control, and Dynamics, vol. 42, no. 5, pp. 1116-1129, May. 2019

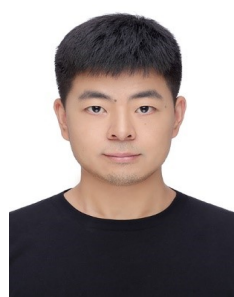

Tuo Han received the B.S. and M.S. degrees in guidance, navigation, and control from the Northwestern Polytechnical University, Xi' an, China, in 2014 and 2017, respectively, and is currently pursuing the Ph.D. degree in control science and engineering from the Beihang University, Beijing, China.

$\mathrm{He}$ is currently a visiting researcher in aerospace with Cranfield University, Cranfield, U.K. His research interests include flight guidance and control of aerospace vehicles, intelligent control and applications.

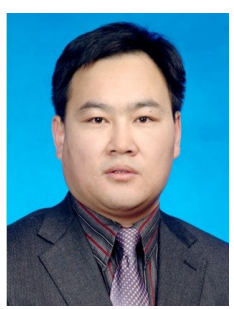

Qinglei Hu (Senior Member, IEEE) received his B.Eng. degree in electrical and electronic engineering from Zhengzhou University, Zhengzhou, China, in 2001, and $\mathrm{Ph} . \mathrm{D}$. degree in guidance and control from Harbin Institute of Technology, Harbin, China, in 2006.

From 2003 to 2014, he had been with the 
Department of Control Science and Engineering at Harbin Institute of Technology. He joined Beihang University, Beijing, China, in 2014, as a full Professor. He worked as a Post-Doctoral Research Fellow in Nanyang Technological University, Singapore, from 2006 to 2007, and 2008 to 2009. He visited the University of Bristol, U.K., as a Senior Research Fellow supported by Royal Society Fellowship. From 2010 to 2011, he visited Concordia University supported by Natural Sciences and Engineering Research Council of Canada. His research interests include guidance, navigation, and control theory and applications. He is an Associate Fellow of American Institute of Aeronautics and Astronautics (AIAA).

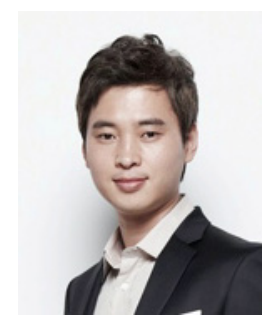

Hyo-Sang Shin received his BSc on aerospace engineering from Pusan National University in 2004 and gained an MSc on flight dynamics, guidance and control in Aerospace Engineering from KAIST and $\mathrm{PhD}$ on cooperative missile guidance from Cranfield University in 2006 and 2010, respectively.

$\mathrm{He}$ is currently a Professor of Guidance, Control and Navigation (GNC) Systems in Autonomous and Intelligent Systems Group at Cranfield University. His current research interests include multiple target tracking, adaptive and sensor-based control, data-centric GNC, and distributed control of multiple agent systems.

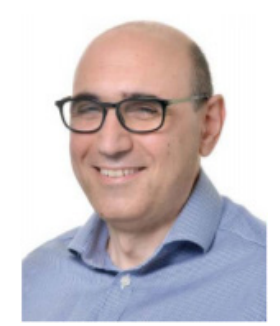

Antonios Tsourdos obtained a MEng on Electronic, Control and Systems Engineering from the University of Sheffield, in 1995, an MSc on Systems Engineering from Cardiff University in 1996 and a PhD on Nonlinear Robust Autopilot Design and Analysis from Cranfield University in 1999. He joined the Cranfield University in 1999 as lecturer, appointed Head of the Centre of Autonomous and Cyber-Physical Systems in 2007 and Professor of Autonomous Systems and Control in 2009 and Director of Research - Aerospace, Transport and Manufacturing in 2015.

He leads the research theme on autonomous systems within the School of Aerospace, Transport and Manufacturing at Cranfield University. He has diverse expertise in both unmanned and autonomous vehicles as well as networked systems. He conducts basic and applied research in the fields of guidance, control and navigation for single and multiple unmanned autonomous vehicles as well as research on cyber physical systems.

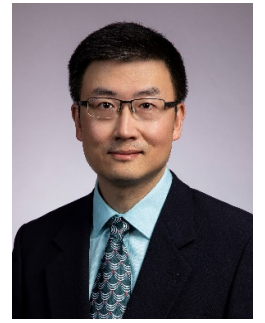

Ming Xin (Senior Member, IEEE) received the B.S. and M.S. degrees in automatic control from the Nanjing University of Aeronautics and Astronautics, Nanjing, China, in 1993 and 1996, respectively, and the Ph.D. degree in aerospace engineering from the Missouri University of Science and Technology, Rolla, MO, USA, in 2002.

$\mathrm{He}$ is currently a Professor with the Department of Mechanical and Aerospace Engineering, University of Missouri, Columbia, MO. His research interests include flight mechanics, guidance, navigation, and control of aerospace vehicles, optimal control theory and applications, estimation/filtering, and cooperative control of multiagent systems. Dr. Xin was the recipient of the U.S. National Science Foundation CAREER Award. He is an Associate Fellow of American Institute of Aeronautics and Astronautics (AIAA), a Senior Member of American Astronautical Society (AAS), and a member of American Society of Mechanical Engineers (ASME). He is an Associate Editor for AIAA Journal of Spacecraft and Rockets and ASME Journal of Dynamic Systems, Measurement, and Control, and a Technical Editor for the IEEE/ASME TRANSACTIONS ON MECHATRONICS. 
$2021-05-14$

\title{
Incremental twisting fault tolerant control for hypersonic vehicles with partial model knowledge
}

\author{
Han, Tuo
}

IEEE

Han T, Hu Q, Shin HS, et al., (2022) Incremental twisting fault tolerant control for hypersonic vehicles with partial model knowledge. IEEE Transactions on Industrial Informatics, Volume 18, Number 2, February 2022, pp. 1050-1060

https://doi.org/10.1109/TII.2021.3080303

Downloaded from Cranfield Library Services E-Repository 